\title{
Effectiveness of Double-hit Model (Post-weaning Social Isolation and NMDA Receptor Antagonist) in the Development of Schizophrenic Like Symptoms on Rodents: A Protocol for a Systematic Review.
}

Khanyiso Bright Shangase (D 210507028@stu.ukzn.ac.za)

University of KwaZulu-Natal - Westville Campus https://orcid.org/0000-0001-9940-1768

Thabo Magwai

University of KwaZulu-Natal - Westville Campus

Fredrick Otieno Oginga

University of KwaZulu-Natal - Westville Campus

Khethelo Richman Xulu

University of KwaZulu-Natal - Westville Campus

Thabisile Mpofana

University of KwaZulu-Natal - Westville Campus

\section{Protocol}

Keywords: Schizophrenia, Animal model, Social isolation, Non-competitive NMDA receptor antagonist

Posted Date: July 1st, 2021

DOI: https://doi.org/10.21203/rs.3.rs-643962/v1

License: (c) (i) This work is licensed under a Creative Commons Attribution 4.0 International License. Read Full License

Version of Record: A version of this preprint was published at NeuroSci on February 9th, 2022. See the published version at https://doi.org/10.3390/neurosci3010009. 


\section{Abstract}

\section{Background}

Schizophrenia is a heterogeneous neuropsychiatric disorder, categorized by positive, negative, and cognitive symptoms. In trying to improve diagnosis and treatment of schizophrenia researchers have turn to "dual hit" models of schizophrenia that are able to reproduce all symptoms of the disorder. The main objective of this protocol is to present a transparent process on how we plan to review the existing international literature on effectiveness of "dual hit" models used to induce schizophrenia on rodents.

Methods

Literature search strategies will be developed using medical search headings (MeSH). The MEDLINE (PubMed), EMBASE, and Google Scholar databases will be used to search for published studies electronically. We will search for studies involving inducing schizophrenic symptoms using "dual hit" rodent model (post-weaning social isolation and NMDA receptor antagonist). Studies will be screened by titles, abstracts, keywords, and synonyms followed by identifying the full-text articles. All studies that will pass the quality assessment will be included. Data will be extracted by two authors independently and in duplicate, from each eligible study in ensuring that there is consistency between reviews. If the design and comparator are sufficiently homogenous for all studies, meta-analysis will be conducted using a random-effect model.

\section{Discussion}

The results of this review will contribute in the development of new "dual hit" models that will be able to characterize schizophrenia symptoms better. It will also shed light to researchers on new developments that need to be made in improving animal models of schizophrenia.

Systematic reviews registration

PROSPERO CRD42021247585

\section{Background}

Schizophrenia is a devastating neuropsychiatric disorder, characterised by intense disruption in thinking which affects perception, language and the sense of self (1). Symptoms are classically divided into three categories: positive, negative and cognitive (2). Although available antipsychotic treatment is highly effective in reducing positive symptoms, they have limited efficacy for negative and cognitive symptoms $(3,4)$. Better understanding of molecular and neuropathological mechanisms underlying schizophrenia will increase chances of finding new therapeutic targets. This process requires formulation and use of robust and reliable animal (rodent) models of schizophrenia using behaviours with translational significance and predictive validity. Currently there is no single rodent model that fully 
reproduces the different schizophrenia symptom profile, therefore researchers have started to investigate "dual hit" models in rodents in order to produce more broad and robust deficits.

\section{Objectives}

The aim of this systematic review is to evaluate the effectiveness of rodent "dual hit" model (postweaning social isolation and NMDA receptor antagonist) of schizophrenia.

- Investigate the production and severity of positive symptoms.

- Investigate the production and severity of negative and cognitive symptoms.

- Investigate the response to treatment (antipsychotics) of rodents with "dual hit" model of schizophrenia.

This systematic review is registered at the International Prospective Register of Systematic reviews (PROSPERO) (CRD42021247585). We will disclose any deviations from this protocol, and we will update the PROSPERO record accordingly.

\section{Methodology}

This systematic review was developed according to the preferred reporting items for systematic review and meta-analysis protocols (PRISMA-P) (5).

\section{Condition}

The condition is schizophrenia as describe by authors using the following:

Population: we will focus on rodent animal models and we will focus on studies that used "dual hit" model (post-weaning social isolation and NMDA receptor antagonist) to induce schizophrenia on rodents. We will exclude studies that only used post-weaning social isolation to induce schizophrenia on rodents. We will exclude studies that only used NMDA receptor antagonist to induce schizophrenia on rodents.

\section{Outcomes}

The primary outcome of this review will be cognitive impairments caused by schizophrenia, down or upregulation of different genes involved in schizophrenia disorder, neurochemical and structural changes related to schizophrenia disorder. The severity of positive, negative and cognitive symptoms will be evaluated. The gender effect will also be investigated by comparing the response of both male and female rodents to the "dual hit" model.

\section{Inclusion criteria}

Studies will be included in this review based on the following criteria: 
All studies that used the "dual hit" rodent model (post-weaning social isolation and NMDA receptor antagonist) to induce schizophrenia will be included. All published studies will be included regardless of sample size, gender, year of publication, studies in other languages will only be included if they can be translated to English using Google translator.

\section{Exclusion criteria}

We will exclude all non-rodent studies, editorials, letters, and case studies. All unpublished animal studies will also be excluded.

\section{Information sources}

Literature search strategies will be developed using medical search headings (MeSH). The MEDLINE (PubMed), EMBASE, and Google Scholar databases will be used to search for published studies electronically. All databases will be searched from inception until 28 February 2021, with the literature search limited to the English language and rodent subjects. To confirm literature saturation, we will scan the reference list of the included studies. We will search the author's individual files to ensure that all the relevant materials have been captured. Lastly, we will circulate a bibliography of the included articles to the systematic review team, as well as to the schizophrenia experts identified by the team.

\section{Search strategy}

The review will be based on quantitative studies; no study design and language or date limits will be applied to the search. Studies in languages other than English that can be translated sufficiently using Google translate will be included. The literature search will be conducted by two independent authors (Khanyiso Bright Shangase and Thabo Magwai), and the third author (Fredrick Otieno Oginga) will be consulted for arbitration. The search will be based on the following keywords and rodent subject heading; "Schizophrenia", "social isolation", "NMDA receptor antagonists", "Animal models".

\section{Interventions}

Of interest are interventions involving inducing schizophrenic symptoms using dual hit rodent model (post-weaning social isolation and NMDA receptor antagonist). Most laboratory studies socially isolate rat and mouse pups by housing them individually from the first day of weaning from their dams, usually on PND21 to PND28. Depending on individual researcher or study the period of isolation varies from 3 weeks to 12 weeks, and the effect of social isolation depend on the period the animals were isolated. There are many available NMDA receptor antagonists that researchers used to induce schizophrenia on rodents, some antagonists are stronger than others. Different researchers use different NMDA receptor antagonists and different dosages to induce schizophrenia depending on what they want to achieve on their individual studies.

\section{Study records}


Results from the literature search will be uploaded to an internet-based software programme that eases collaboration among reviews during the study selection process, termed distiller systematic review (DSR). All references will be imported into the one single endnotes library version X7. Our team will develop and test screening questions and forms for level 1 and level 2 assessments based on the inclusion and exclusion criteria. Citation abstracts and full-text articles will be uploaded along with screening questions to the DSR. Before the formal screening process, the calibration screening exercise will be conducted to pilot and refine the screening questions. Before the start of the review, we will provide training to new team members not familiar with the DSR software and the content area.

\section{Selection process}

The screening studies process will be performed by two independent authors (Khanyiso Bright Shangase and Thabo Magwai) to avoid any inconsistencies in terms of the eligibility of studies. Firstly, studies will be screened by titles, abstracts, keywords and synonyms followed by identifying the full-text articles. Should any disagreements arise between two authors, a third author (Fredrick Otieno Oginga) will screen such studies and agreement will be reached through discussion. We will seek additional information from study authors where necessary to resolve questions about eligibility. All excluded studies will be listed in a table and the reason for exclusion will be recorded. The PRISMA-flowchart will be prepared to document the final selection process. All selected or included studies will be subjected to data collection, critical appraisal, risk and quality assessment.

\section{Data extraction}

Standardised forms and a thorough instruction guidebook will be used to advise specific adapting of an online data abstraction program DSR. Khanyiso Bright Shangase and Thabo Magwai will extract data, independently and in duplicate, from each eligible study in ensuring that there is consistency between reviews. A calibration exercise will be run before the starting of the review. The third author (Fredrick Otieno Oginga) will intervene for arbitration should any disagreements arise. The author and year of publication, the country, sample size, study design, rodent characteristics (strain, age and gender), period of isolation, NMDA receptor antagonist used and dosage, types of control used, symptoms severity and treatment of symptoms if included will be recorded. The main authors of studies will be contacted where inadequate data is provided to acquire enough information. Individual studies may consist of multiple treatment groups such as different dosages of NMDA receptor antagonist or different social isolation periods. To prevent the possibility of introducing bias caused by multiple statistical comparisons with one control group, groups from various arms studies will be combined into a single group.

\section{Risk of bias and quality assessment}

The Downs and Black checklist will be used to test the quality and biases of included studies (6). The checklist is made up of 4 domains: reporting of bias, external validity, internal validity, and selection bias. The checklist comprises 27 questions, with a maximum score of 27. Downs and Black score are divided into 4 categories: excellent (26-27), good (20-25), fair (15-19), and poor (0-14) (7). For all included studies, 
two independent authors will evaluate the quality of each study (Khanyiso Bright Shangase and Thabo Magwai). In case of disagreements, authors will discuss such studies, and the third author (Fredrick Otieno Oginga) will be then adjudicated.

\section{Publication bias and quality of cumulative evidence}

We will test if the selective reporting of outcomes is present (outcome reporting bias), and the presence of small sample bias in the published literature will be evaluated by comparing the fixed effect estimate with the random effect model. The random effect estimate of interventions is mostly favoured than the fixed effect estimate in the presence of small sample bias. in case of 20 studies or more risk rational or mean difference will be used as effect measures for meta-analysis, furthermore, the funnel plots will be produced and Egger's regression test will be used to measure publication bias. Studies will be subgrouped according to geographic location and methodological quality. The quality of evidence for the primary outcomes will be evaluated using the grading of recommendation assessments, development, and evaluation (GRADE) tool (8).

\section{Data synthesis}

If the design and comparator are sufficiently homogenous for all studies, meta-analysis will be conducted using a random-effect model. Dichotomous outcomes will be measured by using a risk ratio (RR) with a $95 \%$ confidence interval $(\mathrm{Cl})$. Previously it has been shown that RR is more intuitive than odds ratios (OR); sometimes, clinicians tend to interpret OR as RR, which results in an overestimation of the effect. Weighted means differences (with $95 \% \mathrm{Cl}$ ) will be used to analyse continuous data; in the event of different measures standardised mean differences $(95 \% \mathrm{Cl})$ will be used. Descriptive presentation will be used for skewed data and non-quantitative data. All included data will be tested in order to determine the unit of randomisation, and if this unit of randomisation is dependable with the unit of analysis. Issues of interest in the analysis of studies with non-standard design, such as studies with multiple treatment groups, cluster randomised trials, and cross-over trials, will be addressed. Interclass correlation coefficient will be adopted to change the results according to the methods explained in the Cochrane Handbook for systematic reviews of intervention for cluster randomised trials. For a cross-over trial, only data from the first phase will be used and guided by the Cochrane Heart Group. For studies with more than two treatments, the additional treatment arm will be presented. The sensitive analysis will be conducted, and the heterogeneity in the randomisation unit will also be acknowledged.

If there is missing data, we will contact the study's original authors to obtain and retrieve the missing data. Important numerical data will be thoroughly appraised. If the missing data is not retrieved, an imputation method will be applied. We will also test for study heterogeneity by specifically looking at animal factors among trials (strain, age, randomisation concealment, blinding of outcome assessment, losses to follow-up, treatment type, and co-interventions). Statistical heterogeneity will be calculated using the Chi $^{2}$ test (significant level: 0.1 ) and $\mathrm{I}^{2}$ statistic (0\% to $40 \%$ might not be important; $30 \%$ to $60 \%$ might represent moderate heterogeneity; $50 \%$ to $90 \%$ might represent substantial heterogeneity; and $75 \%$ 
to $100 \%$ considerably heterogeneity) (9). If the high level of heterogeneity is observed among the trials $\left(I^{2}>50 \%\right.$ or $\left.P<0.1\right)$, the study design and the characteristics in the included studies will be analysed. The sensitivity analysis and subgroup analysis will be used in trying to define and pinpoint exactly the source of heterogeneity. If observed heterogeneity is $\left(\mathrm{I}^{2}>50 \%\right.$ or $\left.\mathrm{P}<0.1\right)$, the random effect model will be used, and while if heterogeneity is substantial, the meta-analysis will not be performed; instead, a quality qualitative narrative summary will be done.

\section{Confidence in cumulative estimates}

Grading of Recommendations Assessment, Development and Evaluation working group methodology will be used to evaluate the quality of evidence for all outcomes. There are four domains of risk of bias: publication bias, consistency, directness, and precision. The quality of evidence will be tested across all these domains. The quality will be arbitrated as high: more research is improbable to change our confidence in the estimate of effect. This will be followed by moderate: more research is likely to have an important influence on our confidence in the estimate of effect and may change the estimate. Then low: more research is very likely to have an essential influence on our confidence in the estimate of effect and may change the estimate and finally very low: very uncertain about the estimate of effect. The quality of evidence for the primary outcomes will be evaluated using the grading of recommendation assessments, development, and evaluation (GRADE) tool.

\section{Discussion}

Schizophrenia is a severe neuropsychiatric disorder affecting approximately $1 \%$ of the population globally $(10,11)$. The symptoms are divided into three groups: positive (conceptual disorganisation, delusions, hallucinations and thought disorder), negative (anhedonia, motivation, avolition, poverty of thought, social withdrawal and poverty of speech) and cognitive symptoms (attention deficits, cognitive flexibility and working memory) $(12,13)$. Negative symptoms and cognitive impairments, still maintain their resistant spectrum against current antipsychotic treatment. Most animal models of schizophrenia tend to replicate characteristics of positive symptoms of schizophrenia, thus neglecting the important aspect of the negative symptoms (11). Drug-induced models (ketamine or PCP) have been commonly used to replicate the negative symptoms. In contrast the developmental model (post-weaning social isolation) has been shown to reproduce the positive symptoms (14-16). To better understand and manage schizophrenia, it is imperative to develop a model that can reproduce the pathological state and most of the symptoms (both negative and positive) if not all. This systematic review aims to evaluate the effectiveness of the rodent "dual hit" model (post-weaning social isolation and NMDA receptor antagonist) of schizophrenia.

\section{Abbreviations}

GRADE: Grading and Recommendations Assessment, Development and Evaluation. 
MeSH: Medical Subjects Headings

PRISMA-P: Preferred reporting items for systematic review and meta-analysis protocols

PROSPERO: Prospective register of systematic reviews

\section{Declarations}

\section{Acknowledgements}

There are no acknowledgements

\section{Ethics approval and consent to participate}

Not applicable

Consent for publication

Not applicable

\section{Competing interests}

The authors declare that they have no competing interests

\section{Source of financial support}

1. National Research Foundation (NRF) Thuthuka.

2. College of health science of the University of KwaZulu-Natal, Developing Research, Innovation, Localisation and Leadership in South Africa (DRILL) Fund.

\section{Role of the financial funder}

The funder will support the collection of data by the original investigators, data management and data analysis. The funder is not involved in the design of the projects, protocol and the analysis plan. The funder will have no input on the interpretation or publication of the study results.

\section{*Corresponding Author:}

Khanyiso Bright Shangase (guarantor of the review)

Email: brightshuku@gmail.com

\section{Author's contribution}

All authors were responsible and accountable to all parts of the works related to the protocol. More specifically, Khanyiso Bright Shangase has the idea. Khanyiso bright Shangase, Thabo Magwai, Fredrick 
Otieno Oginga, Khethelo Richman Xulu and Thabisile Mpofana contributed to conception and design of the protocol. Khanyiso Bright Shangase conducted the first literature searches. Khanyiso Bright Shangase, Thabo Magwai, Fredrick Otieno Oginga, Khethelo Richman Xulu and Thabisile Mpofana contributed in writing the manuscript. Khethelo Richman Xulu and Thabisile Mpofana revised the manuscript and gave the approval to the final version to be published.

\section{Thabisile Mpofana}

Email: mpofana@ukzn.ac.za

\section{Khethelo Richman Xulu}

Email: xuluk2@ukzn.ac.za

\section{Fredrick Otieno Oginga}

221017678@stu.ukzn.ac.za

\section{Thabo Magwai}

Email: thibos.thabo@gmail.com

\section{Acknowledgements}

Not applicable

\section{Affiliations}

1. University of KwaZulu-Natal, College of health science, School of laboratory medicine and medical science, Department of human physiology.

2. University of Kwazulu-Natal, College of health science, Developing Research, Innovation, Localisation and Leadership in South Africa (DRILL).

\section{References}

1. Berry K, Haddock G. The implementation of the NICE guidelines for schizophrenia: barriers to the implementation of psychological interventions and recommendations for the future. Psychol Psychother. 2008;81(Pt 4):419-36.

2. Crow TJ. Molecular pathology of schizophrenia: more than one disease process? Br Med J. 1980;280(6207):66-8.

3. Harvey PD, McClure MM. Pharmacological approaches to the management of cognitive dysfunction in schizophrenia. Drugs. 2006;66(11):1465-73. 
4. Keefe RS. Cognitive deficits in patients with schizophrenia: effects and treatment. J Clin Psychiatry. 2007;68 Suppl 14:8-13.

5. Moher D, Shamseer L, Clarke M, Ghersi D, Liberati A, Petticrew M, et al. Preferred reporting items for systematic review and meta-analysis protocols (PRISMA-P) 2015 statement. Syst Rev. 2015;4(1):1.

6. Downs SH, Black N. The feasibility of creating a checklist for the assessment of the methodological quality both of randomised and non-randomised studies of health care interventions. Journal of epidemiology and community health. 1998;52(6):377-84.

7. Hooper P, Jutai JW, Strong G, Russell-Minda E. Age-related macular degeneration and low-vision rehabilitation: a systematic review. Canadian journal of ophthalmology Journal canadien d'ophtalmologie. 2008;43(2):180-7.

8. Guyatt G, Oxman AD, Akl EA, Kunz R, Vist G, Brozek J, et al. GRADE guidelines: 1. IntroductionGRADE evidence profiles and summary of findings tables. J Clin Epidemiol. 2011;64(4):383-94.

9. Field AP. Meta-analysis of correlation coefficients: a Monte Carlo comparison of fixed-and random-effects methods. Psychol Methods. 2001;6(2):161-80.

10. McGrath J, Saha S, Chant D, Welham J. Schizophrenia: a concise overview of incidence, prevalence, and mortality. Epidemiologic reviews. 2008;30:67-76.

11. Jones C, Watson D, Fone K. Animal models of schizophrenia. British journal of pharmacology. 2011;164(4):1162-94.

12. Andreasen NC. Symptoms, signs, and diagnosis of schizophrenia. Lancet (London, England). 1995;346(8973):477-81.

13. Bosia M, Buonocore M, Bechi M, Santarelli L, Spangaro M, Cocchi F, et al. Improving Cognition to Increase Treatment Efficacy in Schizophrenia: Effects of Metabolic Syndrome on Cognitive Remediation's Outcome. Frontiers in psychiatry. 2018;9:647.

14. Silva-Gómez AB, Rojas D, Juárez I, Flores G. Decreased dendritic spine density on prefrontal cortical and hippocampal pyramidal neurons in postweaning social isolation rats. Brain research. 2003;983(1-2):128-36.

15. Del Arco A, Zhu S, Terasmaa A, Mohammed AH, Fuxe K. Hyperactivity to novelty induced by social isolation is not correlated with changes in D2 receptor function and binding in striatum. Psychopharmacology. 2004;171(2):148-55.

16. Jenkins TA, Harte MK, Reynolds GP. Effect of subchronic phencyclidine administration on sucrose preference and hippocampal parvalbumin immunoreactivity in the rat. Neuroscience letters. 2010;471(3):144-7. 


\section{Supplementary Files}

This is a list of supplementary files associated with this preprint. Click to download.

- PRISMAPchecklist.docx 